American Journal Applied Sciences 5 (11): 1535-1542, 2008

ISSN 1546-9239

(C) 2008 Science Publications

\title{
Amphoteric Starch in Simultaneous Process Preparation with Box-Behnken Design for Optimal Conditions
}

\author{
${ }^{1}$ Sopa Cansee, ${ }^{2}$ Juntanee Uriyapongson, ${ }^{1}$ Commueng Watyotha \\ ${ }^{1}$ Thavachai Thivavarnvongs and ${ }^{3}$ Jatuphong Varith \\ ${ }^{1}$ Department of Agricultural Engineering and ${ }^{2}$ Department of Food Technology, Khon \\ Kaen University, Khon Kaen 40002, Thailand, and ${ }^{3}$ Department of Agricultural and \\ Food Engineering, Maejo University, Chiang Mai 50290, Thailand
}

\begin{abstract}
Cassava starch was chemically modified to produce an amphoteric starch. The amphoteric starch was prepared in a simultaneous process which was evaluated in relation to four factors: temperature $\left(45-55{ }^{\circ} \mathrm{C}\right)$, 3-chloro-2-hydroxypropyltrimethylammonium chloride (CHPTAC, 4-12\%) and sodium tripolyphosphate (STP, 2-8\%) concentrations, and reaction time (2-6 h). The extent of amphoteric starch produced was determined by the degree of substitution (DS) of the nitrogen content and percentage of phosphorus content. In addition, a quantitative response of yield and whiteness as well as pasting characteristics were tested. Response surface analysis showed that the DS (0.01-0.05) increased with increasing temperature, CHPTAC and reaction time. The phosphorus content $(0.09-$ $0.34 \%$ ) followed a parabolic shape with all of the factors. Consequently, the response surface methodology appears to be a powerful technique to determine the optimal conditions for the production of amphoteric starches.
\end{abstract}

Keywords: Amphoteric starch, Box-Behnken design, Response surface methodology

\section{INTRODUCTION}

Cassava starch is an important food ingredient in tropical countries such as Brazil, Nigeria, Indonesia and Thailand. Cassava starch has often been modified, and Thailand is also a country where modified starch from cassava starch is produced in large scale ${ }^{[1]}$. The traditional starch modifications are usually carried out in an aqueous medium and require a high concentration of modifying reagent to achieve the desired degree of chemical binding ${ }^{[2]}$. The commercial amphoteric starches consist of cationic and anionic modifications. Typically, cationic derivations are starch ethers prepared from tertiary amino or quaternary ammonium reagents, whereas anionic agents substitute with phosphate, phosphonate, sulfate, sulfonate or carboxyl groups $^{[3]}$ depending on specific objectives. The main applications of amphoteric starch are in the paper industry as wet-end additives, coating binders, retention aids, and overall dry-strength agents ${ }^{[4]}$. The use of recycled fibre has been growing steadily since 1984 to around $50 \%$ (the recycling rate is calculated on the basis of recovered paper used in recycling compared to total paper consumption) of the fibre mass used in paper production ${ }^{[5]}$. The increased recycling of fibres has decreased the pulp strength of the bonding fibres. The amino groups on the amphoteric molecules create new hydrogen bonds with hydroxyl groups on the cellulose. Thus, if the number of hydrogen bonds is increased, stronger paper is obtained. Therefore, the use of amphoteric starch has been steadily growing alongside with using of recycled fibre in the paper industry.

Preparation of amphoteric starches has employed two-step treatments of starch with cationic and anionic modifying reagents $\left.{ }^{[4,}, 6,7, \quad 8\right]$. The traditional dual treatments of the amphoteric starch preparations are not resource efficient and require heat for reaction and a drying process, water for washing and unnecessary management. The simultaneous process of amphoteric preparation can be of economic benefit. Review of the literature shows that only Youquan et al ${ }^{[9]}$ have studied preparation of phosphate amphoteric starch in a simultaneous process, and its application. The results showed that amphoteric starch has more advantages than cationic starch in the paper industry. It can

Corresponding Author: Juntanee Uriyapongson, Department of Food Technology, Faculty of Technology, Khon Kaen University, Khon Kaen, 40002, Thailand, Tel: +66-43362132, Fax: +66-43362131, 
increase paper strength by more than $17 \%$. However a suitable experimental planning research should be carried out for industrial paper grades. Furthermore, relevant amphoteric starch properties, such as viscosity, should always be considered in paper production.

The aim of this research was to study the preparation of amphoteric starch in a simultaneous process and account for the effects of temperature, reagent concentrations (CHPTAC and STP) and reaction time. A Box-Behnken design $\left.{ }^{[2,} 10,11\right]$ was proposed because this type of design was able to fit a second order model that would be required for optimal condition studies. The empirical model can be useful for effective amphoteric starch preparation. In addition, amphoteric starches were analyzed for the degree of substitution of the cationic derivatives, phosphorus content and pasting properties. Their yield and whiteness were to be tested.

\section{MATERIALS AND METHODS}

Materials: Powdered cassava starch obtained from Sanguan Wongse Industries Co. Ltd., Thailand (the largest volume supplier of modified starch in Asia) was used in this study. The primary properties of starch reported by the company were as follows: whiteness, 93.7\%; pH, 6.0; sulphur dioxide, 27.6 ppm; viscosity, 720 BU and pulp, $0.02 \%$. The CHPTAC and STP were obtained from Sigma-Aldrich Chemical Ltd, Switzerland. Absolute alcohol was $99.8 \%$ pure and distilled water was used. All other reagents used in this work were of analytical grade.

Preparation of amphoteric starches: Amphoteric starch was prepared adapting the procedure of Youquan et al. ${ }^{[9]}$. To a $500 \mathrm{~mL}$ beaker was added $100 \mathrm{~mL}$ of water, $100 \mathrm{~mL}$ of ethanol, $35 \% \mathrm{w} / \mathrm{w}$ of dry cassava starch, then $3.5 \% \mathrm{w} / \mathrm{w}$ of sodium hydroxide compared with dry cassava starch weight with mechanical stirring (Model DLH, Velp Scientifica Srl, Italy) at speed 200 rpm. The beaker was covered with aluminum foil to prevent evaporation of the solution. Table 1 shows the independent and dependent variables. A four-factor, three-level Box-Behnken study design was used. The specific temperatures $\left(45-55{ }^{\circ} \mathrm{C}\right)$ were controlled by water bath (Model WB/OB7-45, Memmert, Germany). STP $(2-8 \%)$ and CHPTAC (4-12\%) by weight of dry cassava starch were slowly added with stirring over 1015 minutes because of their limited solubility. Then, the chemically modified starch was allowed to stand for between 2 and $6 \mathrm{~h}$.

The resulting amphoteric starch product was titrated with hydrochloric acid (3M) to $6.5 \mathrm{pH}$, three-time (with washing in between), centrifuged with Centurion Model 3000 system (Centurion Scientific Ltd, UK) at 1600 rpm for 15 minutes, and air-dried at $50{ }^{\circ} \mathrm{C}$ in an oven overnight. The amphoteric starch yields were weighed and milled with a hammer mill (Model 1090, Tecator, Poland) in the preparation for nitrogen and phosphorus content analysis.

Degree of substitution (DS) and phosphorus content: The DS of a starch derivative, defined as the number of hydroxyl $(\mathrm{OH})$ groups substituted per Dglucopyranosyl structural unit of the starch polymer, had a maximum possible value of $3^{[12]}$. Typically, cationic substitution ranges from 0.01 to 0.05 in commercial wet-end cationic starches for the paper industry $^{[13]}$. Similarly, chemical modification of starches is made to DS values less than $0.1^{[14]}$ that are used as adhesives for paper and paper products. The DS-values of amphoteric starches can be derived from the nitrogen content using the equation below ${ }^{[15]}$. The nitrogen content was determined by the Kjeldahl method $^{[16]}$ using a Kjeltech distilling unit (Model 1026, Tecator, Poland).

$$
\text { Degree of substitution (DS) }=\frac{162 N}{1400-117 N}
$$

where $\mathrm{N}$ is the Kjeltech nitrogen content (\%). The mean value of DS was calculated from three replications. The bond phosphorus content was assayed following the protocol proposed by Smith and Caruso ${ }^{[17]}$. The residual phosphorus was analyzed by a UV/Vis Lambda 2 spectrophotometer (Perkin-Elmer Corp. Norwalk, USA). The phosphorus content (triplicate) was calculated from a standard curve of known phosphorus concentrations, from absorption at $460 \mathrm{~nm}$ using the Beer-Lambert law.

Rapid visco analyser profile: The amphoteric starch products were milled to pass through a $150 \mu \mathrm{m}$ filter using a milling and sieving machine (Model AS200, Retsch, Germany). The moisture contents were determined with a moisture analyzer (Model MB45, OHAUS Corp. New Jersey, USA). The changes in the apparent viscosity of the amphoteric starch samples were measured using an RVA-4 apparatus (NewPort Scientific Pty. Ltd., Warriewood, Australia). A two and a half gram sample, adjusted to $14 \%$ moisture basis, was added to $25 \mathrm{~mL}$ of chilled distilled water already placed in the canister. A plastic paddle was inserted into the canister and it was rotated to disperse the starch 
without any lumps. The canister, along with the paddle, was placed in the RVA and the test was initiated. The sample temperature was equilibrated to $50{ }^{\circ} \mathrm{C}$ and subjected to a heating cycle increasing at a rate of 12 ${ }^{\circ} \mathrm{C} / \mathrm{min}$ to a maximum temperature of $95{ }^{\circ} \mathrm{C}$, holding at $95{ }^{\circ} \mathrm{C}$ for 2.5 minutes, followed by a cooling cycle at the same rate to a minimum temperature of $50{ }^{\circ} \mathrm{C}$. Apparent viscosity and temperature profiles were recorded and monitored through an Acer personal computer. The curves were analysed for peak, trough and final viscosity. The breakdown and setback viscosity were calculated as the difference between initial peak and trough viscosity, and final and trough viscosity, respectively.

Design of experiments: A four-factor, three-level BoxBehnken design was used in this study. This design is suitable for exploration of quadratic response surfaces and construction of a second order polynomial model, thus helping in optimizing a process using a small number of experimental runs. A total of 29 experiments were carried out (Table 2). The design consists of three replicated center points, and a set of six points lying at the midpoints of each edge of the multidimensional cube. The nonlinear computer generated quadratic model $^{[10,18]}$ is given as

$\mathrm{y}=\mathrm{b}_{0}+\sum_{i} b_{i} x_{i}+\sum_{i} b_{i j} x_{i}^{2}+\sum_{j} \sum_{k(k>j)} b_{j k} x_{j} x_{k}$

with $\mathrm{i}=\mathrm{x} 1-\mathrm{x} 4 ; \mathrm{j}=\mathrm{x} 1-\mathrm{x} 3$ and $\mathrm{k}=\mathrm{x} 2-\mathrm{x} 4$, where $\mathrm{y}$ is the response variables, $\mathrm{b}$ is the regression coefficients of the model, and $\mathrm{x}$ is the coded level of an independent variable. The regression equation above was optimized using Design Expert software Version 5.0.8 (Stat-Ease Inc., Minneapolis, USA). The statistical significance of the second order model equation was determined by Fvalue, lack of fit and the multiple coefficient of determination $\left(\mathrm{R}^{2}\right)$.

\section{RESULTS AND DISCUSSION}

In the experimental Box-Behnken procedure, the influences of the four factors (see Table 1) on the DS, the phosphorus content, yield and whiteness of the amphoteric starch were determined (Table 2). Moreover the $\mathrm{pH}$ of solutions in the reactor during experiments was recorded. The $\mathrm{pH}$ of the aqueous medium prepared for the amphoteric starch ranged from 10.30-11.80. Yield (recovery of the modified starch to original material) was $69.6-96.2 \%$. Whiteness of the final products, measured with a Kett C-300-3 apparatus (Kett Electric Laboratory, Tokyo, Japan) was in range of 86.4-92.7\%.

Table 1: Variables in the Box-Behnken design

\begin{tabular}{|c|c|c|c|}
\hline \multirow[b]{2}{*}{ Variable } & \multicolumn{3}{|c|}{ Level Used } \\
\hline & $\begin{array}{l}\text { Low } \\
(-1)\end{array}$ & $\begin{array}{c}\text { Medium } \\
(0)\end{array}$ & $\begin{array}{l}\text { High } \\
(+1)\end{array}$ \\
\hline \multicolumn{4}{|l|}{ Independent Variables } \\
\hline Temperature: $\mathrm{x} 1\left({ }^{\circ} \mathrm{C}\right)$ & 45.0 & 50.0 & 55.0 \\
\hline CHPTAC: x2 (\%ds) & 4.0 & 8.0 & 12.0 \\
\hline STP: x3 (\%ds) & 2.0 & 5.0 & 8.0 \\
\hline Time: x4 (hours) & 2.0 & 4.0 & 6.0 \\
\hline \multicolumn{4}{|l|}{ Dependent Variables } \\
\hline \multicolumn{4}{|l|}{ DS: y1 } \\
\hline \multicolumn{4}{|c|}{ Phosphorus content: y2 (\%) } \\
\hline \multicolumn{4}{|l|}{ Yield: y3 (\%) } \\
\hline Whiteness: y4 (\%) & & & \\
\hline
\end{tabular}

Degree of substitution and phosphorus content: The range of DS values obtained (0.01-0.05) was similar to those usually observed in commercial paper products. The DS increased linearly with increasing temperature and reaction time, whereas it slightly changed with increasing STP. In addition, DS changed in parabolic profile with increasing CHPTAC (Fig. 1); it rapidly increased to a maximal value and then decreased similarly. The statistical analysis indicated significant changes $(P<0.001)$ for temperature and reaction time (Table 3). Furthermore, DS was significant with interaction of CHPTAC and reaction time. The phosphorus content ranged of 0.09 to $0.34 \%$ and showed similar behavior to other factors (Fig. 2). It increased to a maximal value with these factors, then decreased, statistically significant $(P<0.01)$ with CHPTAC and STP. Moreover, the phosphorus content was significant with a quadratic relationship with all of the factors.

Pasting characteristics of amphoteric starches: The viscosity was expressed in SI units of centipoise (cP) which was equivalent to one-tenth rapid viscosity unit (RVU). Figure 3A and 3B show the similar trends of the pasting characteristics of the amphoteric starches with CHPTAC and STP factor as well as temperature and reaction time factor. Peak and breakdown viscosity increased with all increasing factors. Conversely, final and setback viscosity decreased. The peak viscosity ranged from 3554 to $3786 \mathrm{cP}$, indicating the waterbinding capacity of starch, whereas the breakdown 
Table 2: Experimental data and the observed response values used in the Box-Behnken design for the response surface methodology $\mathrm{y}^{\mathrm{a}}$

\begin{tabular}{|c|c|c|c|c|c|c|c|c|}
\hline \multirow[b]{2}{*}{ Run } & \multicolumn{4}{|c|}{ Code levels } & \multicolumn{4}{|c|}{ Experimental data } \\
\hline & $\mathrm{x} 1$ & $\mathrm{x} 2$ & $\mathrm{x} 3$ & $\mathrm{x} 4$ & $\mathrm{y} 1$ & $\mathrm{y} 2$ & $\mathrm{y} 3$ & y4 \\
\hline 1 & 1 & -1 & 0 & 0 & 0.03 & 0.22 & 77.29 & 86.40 \\
\hline 2 & -1 & 0 & -1 & 0 & 0.04 & 0.16 & 89.99 & 89.83 \\
\hline 3 & 0 & -1 & 0 & -1 & 0.02 & 0.29 & 88.02 & 88.67 \\
\hline 4 & 0 & -1 & 0 & 1 & 0.03 & 0.24 & 96.19 & 87.77 \\
\hline 5 & 0 & 0 & 0 & 0 & 0.04 & 0.34 & 81.45 & 90.47 \\
\hline 6 & 1 & 0 & -1 & 0 & 0.05 & 0.19 & 78.46 & 90.10 \\
\hline 7 & 0 & 0 & -1 & -1 & 0.03 & 0.17 & 79.73 & 90.67 \\
\hline 8 & 0 & -1 & 1 & 0 & 0.03 & 0.18 & 80.60 & 89.63 \\
\hline 9 & 0 & 1 & 0 & 1 & 0.05 & 0.17 & 91.00 & 92.67 \\
\hline 10 & 0 & 0 & -1 & 1 & 0.04 & 0.13 & 74.74 & 91.93 \\
\hline 11 & 0 & -1 & -1 & 0 & 0.03 & 0.21 & 83.70 & 88.63 \\
\hline 12 & 0 & 1 & 0 & -1 & 0.01 & 0.12 & 83.76 & 90.90 \\
\hline 13 & 0 & 0 & 0 & 0 & 0.04 & 0.28 & 89.75 & 89.00 \\
\hline 14 & 0 & 0 & 1 & -1 & 0.03 & 0.29 & 83.05 & 89.43 \\
\hline 15 & 1 & 1 & 0 & 0 & 0.03 & 0.15 & 87.55 & 91.73 \\
\hline 16 & 1 & 0 & 0 & -1 & 0.04 & 0.15 & 91.53 & 89.43 \\
\hline 17 & 0 & 1 & 1 & 0 & 0.02 & 0.28 & 85.92 & 92.17 \\
\hline 18 & 1 & 0 & 1 & 0 & 0.05 & 0.11 & 69.59 & 91.67 \\
\hline 19 & 0 & 0 & 0 & 0 & 0.04 & 0.30 & 93.00 & 89.60 \\
\hline 20 & 1 & 0 & 0 & 1 & 0.05 & 0.13 & 72.36 & 91.77 \\
\hline 21 & 0 & 0 & 0 & 0 & 0.04 & 0.25 & 77.96 & 91.17 \\
\hline 22 & -1 & -1 & 0 & 0 & 0.03 & 0.25 & 93.08 & 89.73 \\
\hline 23 & -1 & 1 & 0 & 0 & 0.02 & 0.09 & 91.64 & 91.53 \\
\hline 24 & 0 & 0 & 0 & 0 & 0.04 & 0.34 & 85.53 & 90.50 \\
\hline 25 & -1 & 0 & 1 & 0 & 0.03 & 0.25 & 95.10 & 90.50 \\
\hline 26 & 0 & 1 & -1 & 0 & 0.03 & 0.09 & 89.26 & 91.10 \\
\hline 27 & 0 & 0 & 1 & 1 & 0.04 & 0.31 & 80.06 & 92.23 \\
\hline 28 & -1 & 0 & 0 & 1 & 0.04 & 0.22 & 92.18 & 90.87 \\
\hline 29 & -1 & 0 & 0 & -1 & 0.03 & 0.21 & 93.49 & 89.87 \\
\hline
\end{tabular}

${ }^{\mathrm{a} A \text { Abreviations: see Table } 1 .}$.

viscosity increased from 1915 to $2296 \mathrm{cP}$. The increase of peak and breakdown viscosity might be due to crosslinking effect. Cross-linking reinforces the hydrogen bonds in a granule with chemical bonds acting as bridges between molecules. Through reinforcement of the granules with chemical bonds which do not rupture on being heated with water, the starch granules are strengthened to resist rupturing and imparting during heating ${ }^{[3]}$. The cross-linking of amphoteric starch might be formed from STP under alkaline conditions $(\mathrm{pH}$ 10.3-11.8). These results corresponded with those of other investigators who prepared cross-linked starches ${ }^{[2,}$ $19,20,21]$. As the mixture subsequently cools, reassociation between starch molecules occurs. Viscosity of the gel formed during and after heating is also an important factor ${ }^{[22]}$. The final viscosity of amphoteric starch samples ranged from 2100 to $2422 \mathrm{cP}$ and the setback viscosity from 610 to $782 \mathrm{cP}$. The final viscosity decreased with increasing factors which indicated decreasing retrogradation of the gels on cooling. Similarly, the low setback viscosity of the starches is due to the low amount of linear molecules released from the granules. The results are useful in selecting the suitable starch as a sizing agent in papermaking. Generally, a starch with low viscosity is preferable because it can be heated at higher concentration and this decreases the amount of water in the process ${ }^{[23]}$.

Regression coefficients: Table 3 summarizes the estimated regression coefficients for the quadratic models fitted to the experimental results and their significances, together with the corresponding $\mathrm{R}^{2}$ values. The adequacy of the model was tested by the lack of fit (adequate prediction of the response within 
the design space) and the $\mathrm{R}^{2[18]}$. The lack of fit is not significant, and the quadratic model seems to adequately fit the response. The coefficient of determination revealed from the models for the DS and phosphorus content responses, were 0.94 and 0.82 . Figure 4A and 4B show a parity plot of the DS and phosphorus content obtained with the empirical model. The experimental and the modelled DS and phosphorus

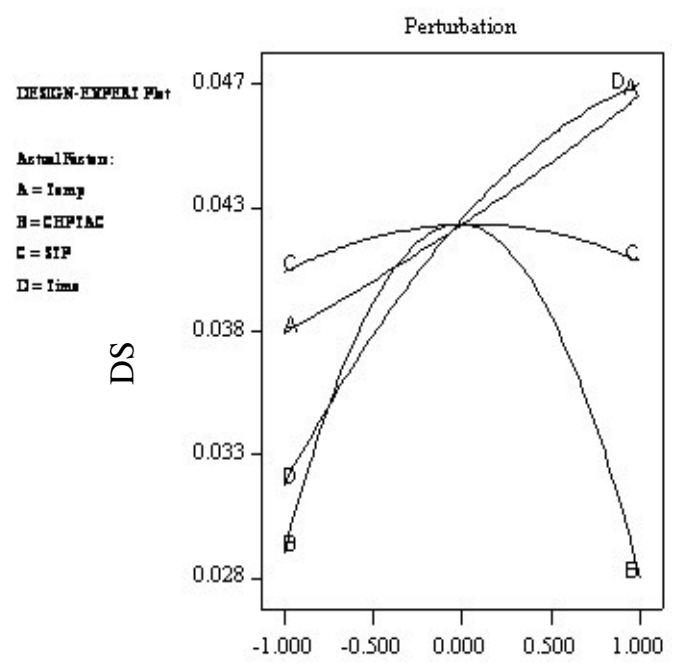

Factor Range in Code Values

Fig. 1: Perturbation plot the DS versus the factors in code values.

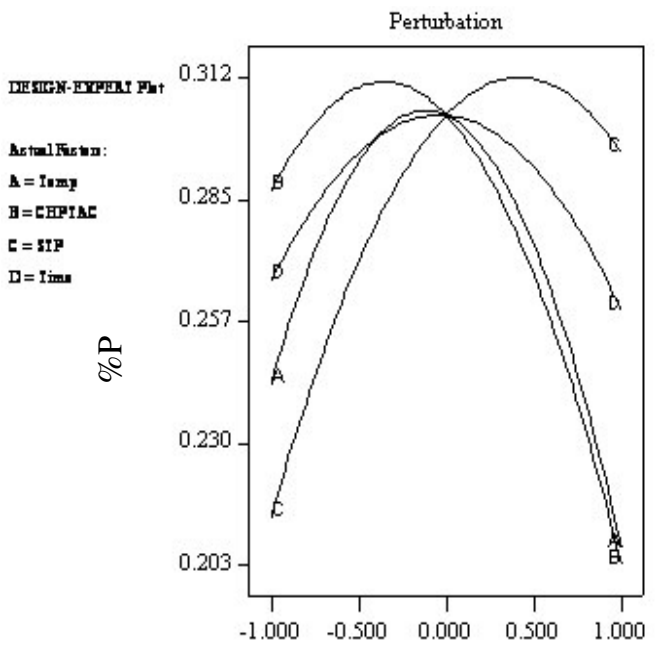

Factor Range in Code Values

Fig. 2: Perturbation plot the phosphorus content versus the factors in code values. content are in good agreement and the results are always greater than zero. The deviation between the experimental and modelled values for phosphorus content is larger than that for the DS.

The relationship of the regression coefficients of the DS was significant with a linear model for $\mathrm{x} 1$ (temperature) and $\mathrm{x} 4$ (reaction time) and a quadratic model for $\mathrm{x} 2$ (CHPTAC) with an interaction between $\mathrm{x} 2$ and $\mathrm{x} 4$. Reaction time gave the largest value $\left(5.557 \times 10^{-3}\right)$ for the coefficient of the DS. It indicated that the longer the reaction time, the higher the DS, whereas the phosphorus content was significant with a linear model of $\mathrm{x} 2$ and $\mathrm{x} 3$ (STP) and with a quadratic relationship of $\mathrm{x} 1, \mathrm{x} 2, \mathrm{x} 3$ and $\mathrm{x} 4$, and also with an interaction between $x 2$ and $x 3$. The coefficient of STP concentration was of moderate significance and was the largest value (0.158) that affected the phosphorus content.

Table 3: Results for the regression coefficients of the quadratic models

\begin{tabular}{lll}
\hline $\begin{array}{l}\text { Regression } \\
\text { equation } \\
\text { coefficients }\end{array}$ & \multicolumn{2}{c}{ Response variables } \\
\cline { 2 - 3 } & & $\begin{array}{l}\text { Phosphorus } \\
\text { content }\end{array}$ \\
\hline $\mathrm{b}_{1}$ & 0.039 & -7.792 \\
Linear & & 0.314 \\
$\mathrm{~b}_{1}$ & $-2.040 \times 10^{-3^{* * *}}$ & $-4.927 \times 10^{-2^{* *}}$ \\
$\mathrm{~b}_{2}$ & $2.145 \times 10^{-3}$ & $0.158^{* * *}$ \\
$\mathrm{~b}_{3}$ & $1.298 \times 10^{-3}$ & $7.968 \times 10^{-2}$ \\
$\mathrm{~b}_{4}$ & $5.557 \times 10^{-3 * * *}$ & \\
Quadratic & & $-3.110 \times 10^{-3 * * *}$ \\
$\mathrm{~b}_{11}$ & $1.700 \times 10^{-5}$ & $-3.640 \times 10^{-3 * *}$ \\
$\mathrm{~b}_{22}$ & $-8.560 \times 10^{-4 * * *}$ & $-5.410 \times 10^{-3 *}$ \\
$\mathrm{~b}_{33}$ & $-1.900 \times 10^{-4}$ & $-9.950 \times 10^{-3 *}$ \\
$\mathrm{~b}_{44}$ & $-6.600 \times 10^{-4}$ & \\
Interaction & & $1.250 \times 10^{-3}$ \\
$\mathrm{~b}_{12}$ & $1.660 \times 10^{-4}$ & $-2.700 \times 10^{-3}$ \\
$\mathrm{~b}_{13}$ & $3.700 \times 10^{-5}$ & $-7.600 \times 10^{-3}$ \\
$\mathrm{~b}_{14}$ & $-5.600 \times 10^{-5}$ & $4.447 \times 10^{-3 *}$ \\
$\mathrm{~b}_{23}$ & $-8.200 \times 10^{-5}$ & $3.085 \times 10^{-3}$ \\
$\mathrm{~b}_{24}$ & $8.950 \times 10^{-4 * * *}$ & $2.306 \times 10^{-3}$ \\
$\mathrm{~b}_{34}$ & $-1.300 \times 10^{-4}$ & 0.0044 \\
$P$-value & 0.0001 & 1.360 \\
Lack of fit & 2.410 & 0.8154 \\
$\mathrm{R}^{2}$ & 0.9435 & \\
\hline
\end{tabular}

${ }^{*}$ Significance at the 0.05 level $(P<0.05)$.

${ }^{* *}$ Significance at the 0.01 level $(P<0.01)$.

**** Significance at the 0.001 level $(P<0.001)$. $\mathrm{b}_{0}$-constant; $\mathrm{b}_{1}$-coefficient of temperature; $\mathrm{b}_{2}$-coefficient of CHPTAC; $\mathrm{b}_{3}$-coefficient of STP; $\mathrm{b}_{4}$-coefficient of reaction time. 
Table 4: Optimum reaction conditions for the production of amphoteric starch with a DS of 0.04 and $\mathrm{P}$ of $0.15 \%$

\begin{tabular}{lll}
\hline Response & Required & Optimum \\
\hline DS & 0.04 & 0.04 \\
Phosphorus content $(\%)$ & 0.15 & 0.15 \\
Peak viscosity $(\mathrm{cP})$ & Minimum & 3653.60 \\
Yield $(\%)$ & $>85.0$ & 91.74 \\
Whiteness $(\%)$ & In range & 90.49 \\
Factor & Code & Level \\
Temperature $\left({ }^{\circ} \mathrm{C}\right)$ & $\mathrm{x} 1$ & 45.00 \\
CHPTAC $(\% \mathrm{ds})$ & $\mathrm{x} 2$ & 8.12 \\
STP $(\% \mathrm{ds})$ & $\mathrm{x} 3$ & 2.80 \\
Time (hours) & $\mathrm{x} 4$ & 4.58 \\
\hline
\end{tabular}

ds: dry cassava starch w/w.
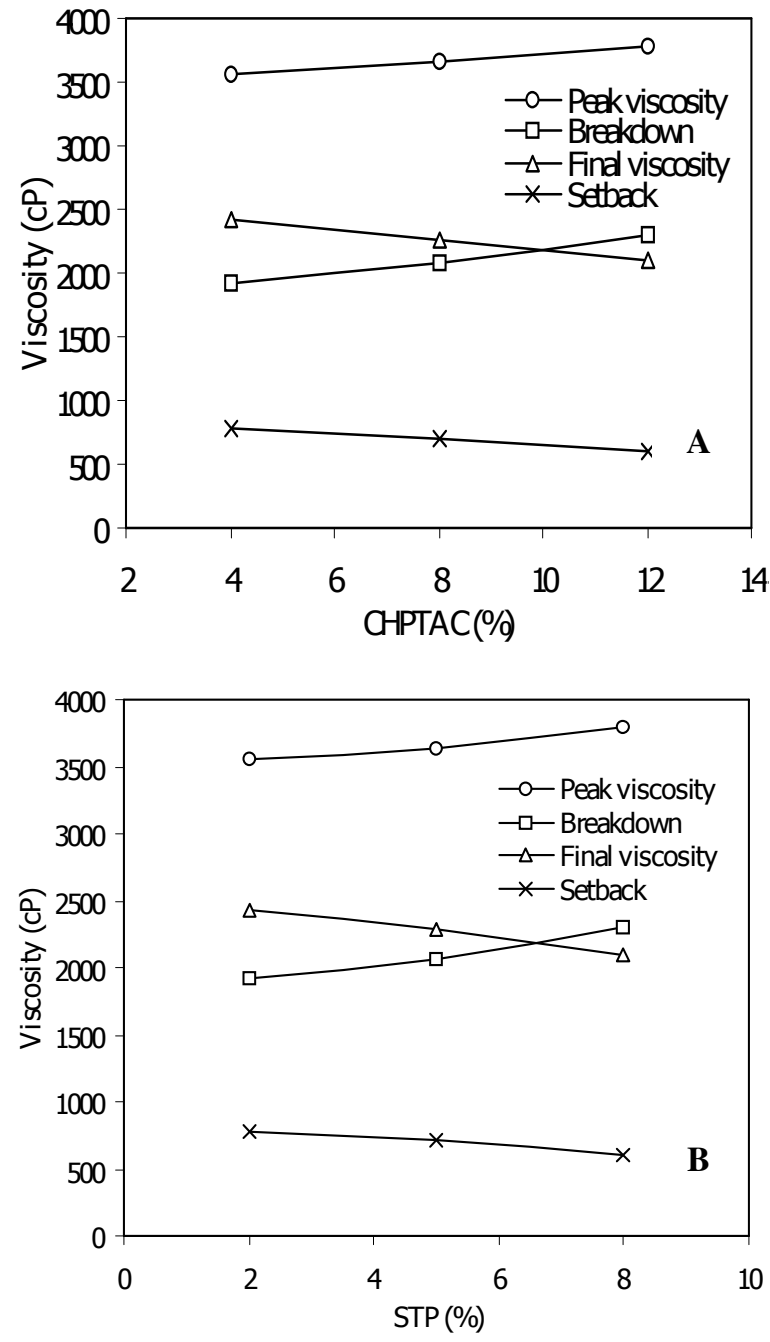

Fig. 3: Effected factors, (A) CHPTAC and (B) STP on peak, breakdown, final and setback viscosity.
Optimization of the responses: The optimization might have one of three meanings: to maximize, to minimize or to set a target of a specific level for factors. The objective is to produce an amphoteric starch with significant DS and phosphorus content, because the amphoteric starch grades would be applied in the different functions of the paper making process.
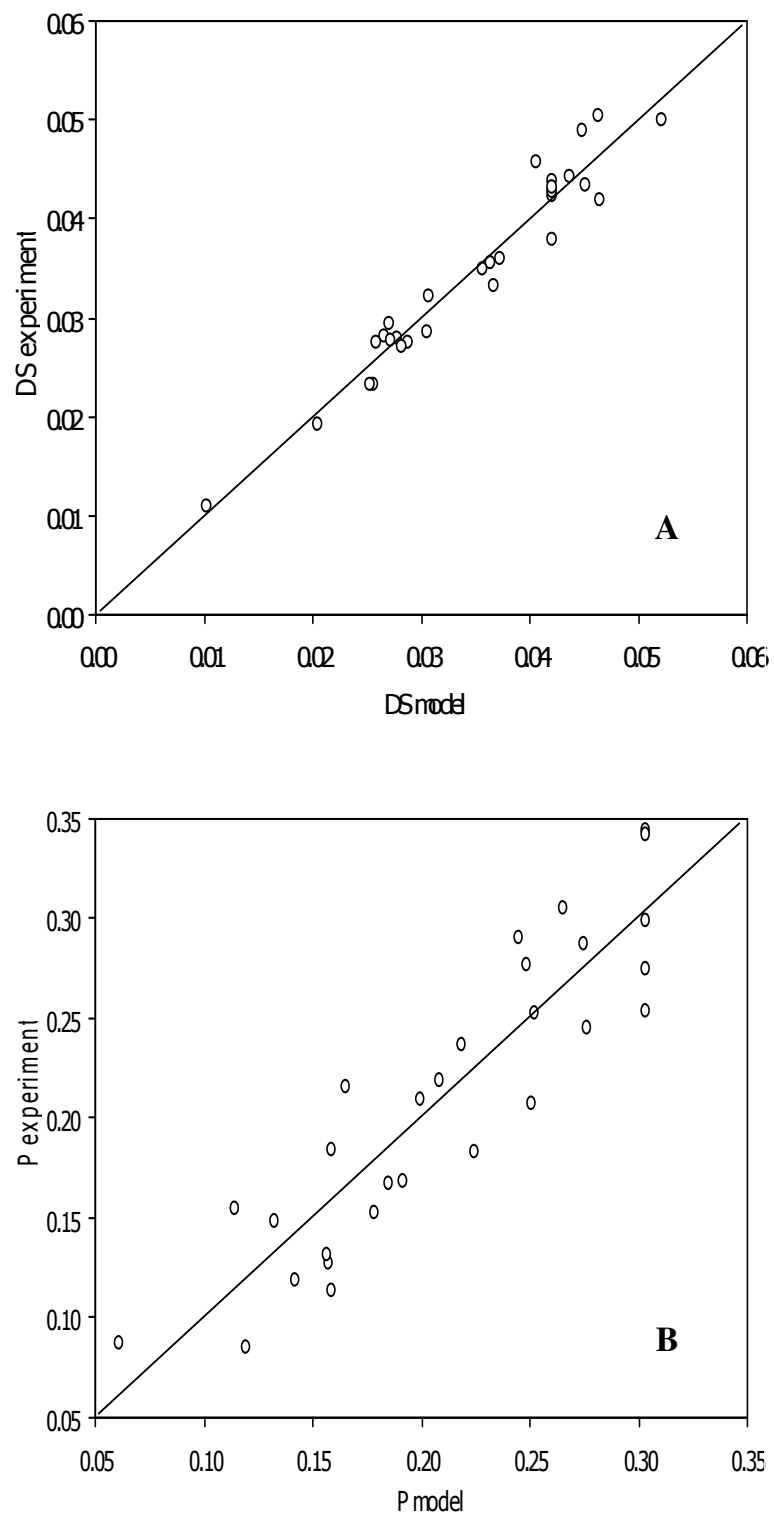

Fig. 4: Observed values of (A) degree of substitution and (B) phosphorus content versus predicted values given by the model. 
The strongest dependency on the DS and phosphorus content is observed in Fig. 1 and 2. The behaviors of both the DS and phosphorus content are confirmed by the empirical model (Table 3). The optimal conditions could be determined with the response surface strategy and numerical optimization solutions. Table 4 shows an example of conditional design of amphoteric starch for a DS equal to 0.04 , phosphorus content equal to $0.15 \%$, peak viscosity of minimum value, yield higher than $85 \%$ and whiteness bounded in the range. The results for the factors were given in the lower half of the table. The temperature, CHPTAC, STP and reaction time were $45.0^{\circ} \mathrm{C}, 8.12 \%$, $2.80 \%$ and $4.58 \mathrm{~h}$, respectively. Correspondingly, the predicted outcome from the model is 0.04 for DS, $0.15 \%$ for phosphorus content, $3653.6 \mathrm{cP}$ for peak viscosity, $91.7 \%$ for yield, and $90.5 \%$ for whiteness respectively. The optimization of responses is an advantage for both pilot studies and manufacturing process. Those responses and conditions are important guides for specific productions, which correspond to their applications.

\section{CONCLUSION}

The results obtained from this research showed that it was possible to produce amphoteric starch by a simultaneous process. The DS and phosphorus content were $0.01-0.05$ and $0.09-0.34 \%$ respectively. The DS was affected by temperature and reaction time, with a quadratic relationship of CHPTAC and an interaction between CHPTAC and reaction time. The phosphorus content was affected by CHPTAC and STP, with a quadratic relationship of all the factors, and an interaction between CHPTAC and STP. The pasting characteristics of the amphoteric starches showed increasing peak and breakdown viscosity with all the process factors. Moreover, the experimental design and response surface methodology appeared to be powerful techniques to determine the optimal conditions for the production of amphoteric starches. The empirical model of the DS and phosphorus content was important prediction tool for amphoteric starch production.

\section{ACKNOWLEDGEMENTS}

The authors would like to express their sincere thanks to the Postgraduate Education and Research Development Project in Postharvest Technology and the Graduate Faculty for financial support, and the Department of Food Technology, Khon Kaen University, Thailand for providing the Kjeltech and spectrophotometer for the measurements of nitrogen and phosphorus contents.

\section{REFERENCES}

1. Sriroth, K., K. Piyachomwan, K. Sangseethong and C. Oates, 2002. Modification of cassava starch. Paper presented $X$ International Starch Convension, Cracow, Poland, 11-14 June 2002.

2. Nabeshima, E. H. and M. V. E. Grossmann, 2001. Functional properties of pregelatinized and crosslinked cassava starch obtained by extrusion with sodium trimetaphosphate. Carbohydr. Polym. 45: 347-353.

3. Wurzburg, O. B., 1986. Modified starches: properties and uses. CRC Press, Inc., Boca Raton, Florida, pp: 3-77.

4. Solarek, D. B., T. A. Dirscherl, H. R. Hernandez and W. Jarowenko, 1989. Amphoteric starches and process for their preparation. US Patent 4876336.

5. Forest-based industries, 2001. Pulp and paper manufacturing. Available from:

http://europa.eu.int/comm/enterprise/forest_based/ pulp_en.html (Last accessed 03/07/07).

6. Hubbard, E. D., R. D. Harvey and M. L. Hogen, 1986. Starch phosphates and amphoteric starch phosphates. US Patent 4566910.

7. Suc, S., J. Defaye, A. Gadelle and J. Kervennal, 1995. Amphoteric starch containing carboxyl and cationic groups. US Patent 5417755.

8. Bindzus, W. and P. A. Altieri, 2002. Amphoteric starches used in papermaking. US Patent 6365002.

9. Youquan, Z., T. Zhangfa and Z. Bengshan, 2003. Preparation of phosphate amphoteric starch in simultaneous process and its application. Chem. J. Internet, 5: 34-40. (in Chinese)

10. Tijseu, C. J., H. J. Scherpenkate, E. J. Stamhuis and A. A. C. M. Beenackers, 1999. Optimisation of the process conditions for the modification of starch. Chem. Eng. Sci., 54: 2765-2772.

11. Kadirgama, K. and K. A. Abou-El-Hossein, 2005. Force prediction model for milling 618 strainless steel using response surface methodology. Am. J. Applied Sci., 2(8): 1222-1227.

12. Fang, J. M., P. A. Fowler, J. Tomkinson and C. A. S. Hill, 2002. The preparation and characterisation of a series of chemically modified potato starches. Carbohydr. Polym., 47: 245-252.

13. Wilke, O. and P. Mischnick, 1995. Analysis of cationic starches: determination of the substitution pattern of $O$-(2-hydroxy-3-trimethylammonium) propyl ethers. Carbohydr. Res., 275: 309-318. 
14. Whistler, R. L. and J. N. BeMiller, 1997. Carbohydrate chemistry for food scientists. Eagan Press, Inc., St Paul, Minnesota.

15. Carr, M. E. and M. O. Bagby, 1981. Preparation of cationic starch ether: a reaction efficiency study. Starch, 33: 310-312.

16. AOAC, 1990. Association of Official Analytical Chemists. Official Methods of Analysis. 15th Edition. Vol. I\&II. Arlington, VA.

17. Smith, R. J. and J. L. Caruso, 1964. Determination of phosphorus. In R. L. Whistler, R. J. Smith, J. N. BeMiller, \& M. L. Wolfrom, (Eds), Methods in carbohydrate chemistry: Vol. 4 (pp. 42-46). Academic Press, New York.

18. Rezzoug, S. A., Z. Maache-Rezzoug, J. Mazoyer, M. Jeannin and K. Allaf, 2000. Effect of instantaneous controlled pressure drop process on the hydration capacity of scleroglucan: optimisation of operating conditions by response surface methodology. Carbohydr. Polym., 42: 7384.
19. Jarowenko, W., 1971. Process for the inhibition of granular starch. US Patent 3553195.

20. Rubens, R. W., 1980. Process for preparing crosslinked starches using STMP. US Patent 4219646.

21. Yook, C., U. H. Pek and K. H. Park, 1993. Gelatinization and retrogradation characteristics of hydroxypropylated and cross-linked rices. J. Food Sci., 58: 405-407.

22. Aryee, F. N. A., I. Oduro, W. O.Ellis and J. J. Afuakwa, 2006. The physicochemical properties of flour samples from the roots of 31 varieties of cassava. Food Control, 17: 916-922.

23. Naing, M. T., 1998. Use of modified tapioca starch in papermaking. M.Sc. thesis, School of Environment, Resources and Development, Asian Institute of Technology, Bangkok, Thailand. 